\title{
Inovações de Processo e Tecnologia no Desenvolvimento de Software
}

\author{
Cristina Cerdeiral, Ana Regina Rocha
}

COPPE/UFRJ - Universidade Federal do Rio de Janeiro

Caixa Postal 68511 - CEP 21945-970 - Rio de Janeiro, Brasil

\{cerdeiral, darocha\}@cos.ufrj.br

\begin{abstract}
This paper describes the state of the art about process and technology innovations in software development and the results obtained in a study based in systematic reviews about this subject. Based on the necessities identified by the study, an environment is presented as a portal for helping in knowledge transfer and approximation between the academy and the industry, besides providing support information for innovating in software development.
\end{abstract}

Resumo. Este artigo descreve o estado da arte sobre inovações de processo e tecnologia no desenvolvimento de software e os resultados obtidos em um estudo baseado em revisão sistemática sobre o tema. Com base nas necessidades identificadas pelo estudo, é apresentado um ambiente no formato de portal que visa auxiliar na transferência de conhecimento e aproximação entre a academia e a indústria, além de fornecer informações de apoio para a inovação no desenvolvimento de software.

\section{Introdução}

A inovação aborda a criação e o desenvolvimento de novas ideias e soluções, transformando oportunidades em utilização prática [Tidd e Bessant, 2009]. Uma inovação é a implementação de um produto (bem ou serviço), ou processo, ou método de marketing, ou método organizacional nas práticas de negócios, local de trabalho ou relações externas da organização, sendo este novo ou significativamente melhorado [OECD e Eurostat, 2005]. A implantação efetiva de inovações é reconhecida como uma forma de construir vantagem competitiva sustentável e aumentar o desempenho organizacional [den Hengst et al., 2004; Aversano et al., 2005; Han e Kang, 2007; Khazanchi et al., 2007; Koc e Ceylan, 2007; Brad et al., 2008].

$\mathrm{Na}$ área de Engenharia de Software, a crescente demanda por software e o aumento da complexidade dos sistemas têm levado à criação de inovações, como novos paradigmas, métodos e ferramentas de desenvolvimento, buscando produzir softwares com maior qualidade e em menor tempo [Agarwal e Prasad, 2000; Woo et al., 2006a]. Estas inovações de processo são novas formas de construir software, e variam no grau com o qual alteram o processo de desenvolvimento de software [Agarwal e Prasad, 2000]. Uma das grandes dificuldades na área é a adaptação às rápidas mudanças, tanto nos paradigmas, métodos e ferramentas de desenvolvimento, como nas necessidades de negócio do mercado e no papel que o software possui nas organizações [Straub e Watson, 2001]. Poucos profissionais presenciam mudanças tão rápidas em suas bases de conhecimento e requisitos de trabalho como os da área de Engenharia de Software. 
A implantação de melhorias inovadoras de processo se encontra nos níveis mais altos nos modelos de maturidade MR - MPS [SOFTEX, 2011] e CMMI [SEI, 2010], pois nestes níveis os processos críticos para as organizações se encontram sob controle estatístico e seus desempenhos são conhecidos e controlados. Desta forma, o impacto das melhorias inovadoras no desempenho dos processos pode ser analisado e controlado [SOFTEX, 2009; SEI, 2010]. Objetivos quantitativos para a melhoria da qualidade e desempenho dos processos críticos para a organização devem ser definidos com base nos objetivos e estratégias de negócio da organização e no conhecimento do desempenho dos processos [Rifkin, 2001; SOFTEX, 2009; SEI, 2010]. A implantação de inovações de processo efetivas deve melhorar a eficiência e a capacidade de resposta da organização, sendo determinante para o crescimento organizacional [Damanpour e Gopalakrishnan, 2001], auxiliando no alcance dos objetivos quantitativos para a melhoria da qualidade e desempenho dos processos críticos para a organização, de forma mensurável [Rifkin, 2001; ISO/IEC, 2002; SOFTEX, 2009; SEI, 2010].

No entanto, identificar potenciais inovações, selecionar as adequadas e implantálas de forma confiável, buscando manter a estabilidade dos processos, obter a melhoria da qualidade e o retorno do investimento é uma atividade complexa, pois envolve realizar mudanças nos processos, impactando em seus desempenhos. Inovações são implantadas para resolver problemas específicos nos processos, porém, se realizadas de forma inadequada, prejudicam a organização em vez de atingir a melhoria desejada, gerando efeitos colaterais [Kuilboer e Ashrafi, 2000].

A inovação não acontece por acidente, necessitando de recursos e processos para facilitar a sua ocorrência [Astebro, 2002]. Na literatura são identificadas as seguintes etapas do processo de gerência de melhorias, envolvendo inovações de processos e de tecnologia [Levine, 2001; Narayana, 2005; Lind, 2006; Tidd e Bessant, 2009; SEI, 2010; SOFTEX, 2011]: (i) Definição dos objetivos de qualidade com base nos objetivos estratégicos e de negócio da organização; (ii) Coleta de sugestões de melhoria no ambiente interno à organização; (iii) Monitoração de inovações existentes no ambiente externo à organização; (iv) Análise das sugestões de melhoria com relação ao alcance dos objetivos de qualidade; (v) Seleção de uma ou mais sugestões de melhoria para implantação; (vi) Planejamento da implantação da sugestão de melhoria selecionada; (vii) Implantação e monitoração da implantação da melhoria selecionada; e (viii) Avaliação dos resultados da implantação da melhoria selecionada no alcance dos objetivos de qualidade definidos.

Este artigo apresenta, na seção 2, os resultados de um estudo baseado em revisão sistemática realizado para identificar o estado da arte em inovações de processo e tecnologia na área de Engenharia de Software. Com base nos resultados do estudo, foram identificadas as etapas do processo de gerência de melhorias inovadoras e incrementais que necessitam de mais pesquisas e contribuições. Para auxiliar em algumas destas etapas, foi construído um ambiente no formato de portal que visa auxiliar na transferência de conhecimento e aproximação entre a academia e a indústria, além de fornecer informações de apoio para a inovação no desenvolvimento de software. Este ambiente é descrito na seção 3. Os autores esperam que a apresentação do ambiente e de seus objetivos estimule a utilização deste pela academia, provendo informações adicionais sobre inovações realizadas na academia para a indústria. As considerações finais são realizadas na seção 4. 


\section{Estudo Baseado em Revisão Sistemática}

Com o objetivo de identificar o estado da arte em inovações de processo e tecnologia na área de Engenharia de Software, foi realizado um estudo baseado em revisão sistemática da literatura, também chamado de mapeamento sistemático, com o foco em inovações de processo de software. A aplicação da revisão sistemática da literatura estabelece um processo formal e controlado para conduzir revisões da literatura, evitando a introdução de tendências que possam desvirtuar os resultados da pesquisa e integrando estudos experimentais para criar generalizações. A sua aplicação requer que seja seguido um conjunto bem definido e sequencial de passos metodológicos segundo um protocolo de pesquisa desenvolvido apropriadamente [Biolchini et al., 2005].

No entanto, neste estudo não é utilizado todo o rigor original proposto por esta técnica, e sim alguns conceitos básicos, não com o intuito de confirmar hipóteses, mas apenas de garantir uma boa cobertura para a pesquisa. O estudo baseado em revisão sistemática seguiu o processo definido em [Montoni, 2007] que pode ser observado na figura 1 e que possui três atividades:

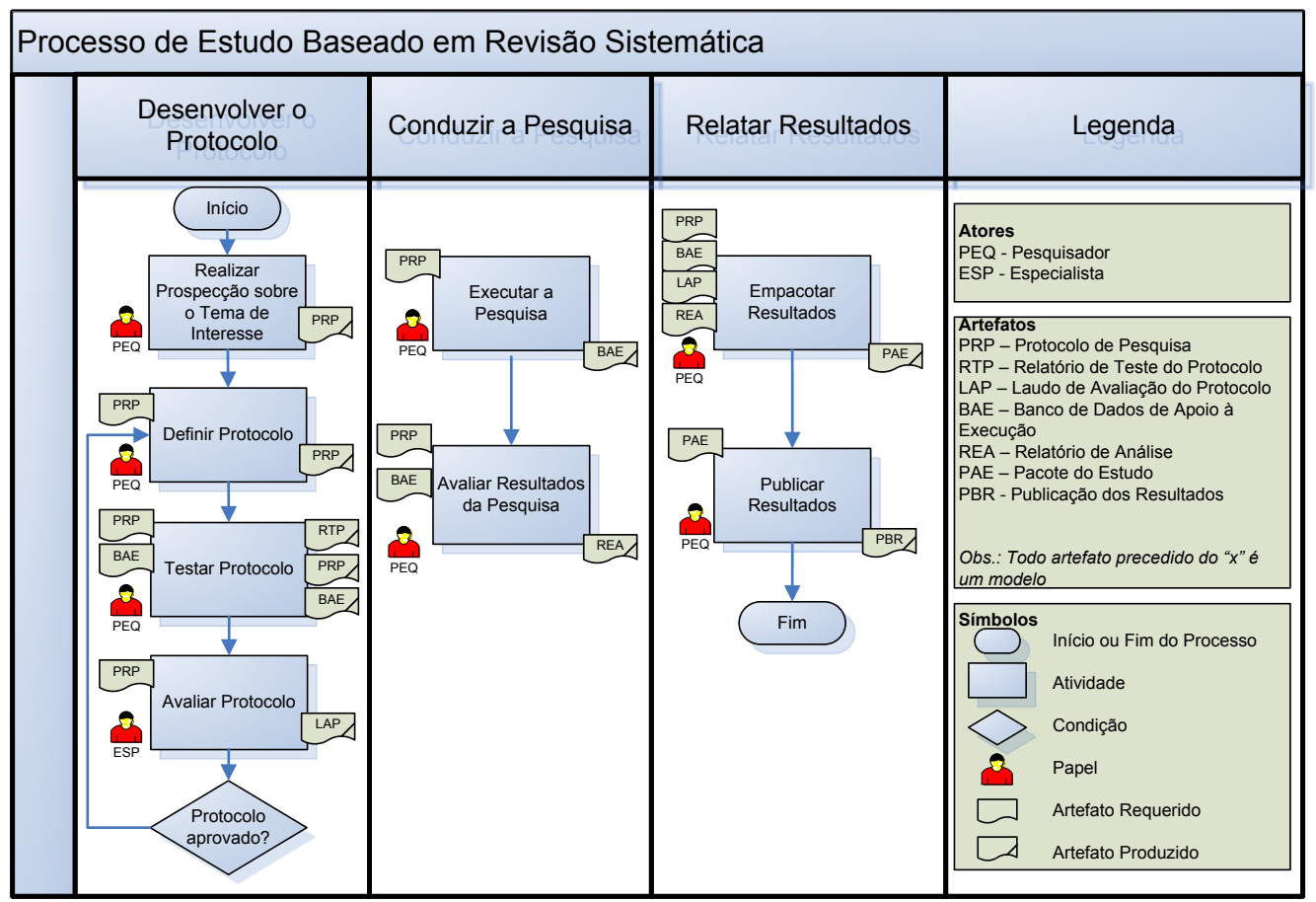

Figura 1. Processo de Estudo Baseado em Revisão Sistemática [Montoni, 2007]

(i) Desenvolver o Protocolo: O pesquisador realiza uma prospecção sobre o tema de interesse, define um protocolo de pesquisa para guiar a condução do estudo, testa e avalia o protocolo. O protocolo é testado para verificar a viabilidade de sua execução, bem como para identificar os ajustes necessários.

(ii) Conduzir a Pesquisa: O estudo é conduzido com base no protocolo e os resultados da pesquisa são avaliados. Esta atividade envolve, também, a realização de análises quantitativas e qualitativas com base nos dados coletados. 
(iii) Relatar Resultados: Os resultados são empacotados e publicados em alguma conferência, revista ou biblioteca de trabalhos científicos.

\subsection{Protocolo de Pesquisa}

\section{$\underline{\text { Objetivo }}$}

O objetivo da pesquisa é identificar os estudos e apoios ou contribuições encontrados na literatura especificamente na área de inovação de processo de software, caracterizando o estado da arte deste tema. Este objetivo foi delineado a partir do paradigma GQM [Basili et al., 1994], conforme descrito a seguir:

Analisar relatos de experiência e publicações científicas sobre inovação e processos de software

Com o propósito de detalhar os estudos e apoios ou contribuições realizadas em inovação de processo de software

Com relação ao tipo de estudo, apoio ou contribuição realizada

Do ponto de vista de pesquisadores

No contexto acadêmico e industrial

Questões de Pesquisa

A seguinte questão foi definida para atender o objetivo da pesquisa:

Quais são os estudos e apoios ou contribuições realizadas em inovação de processo de software nos últimos anos?

A partir da identificação dos estudos e apoios ou contribuições realizadas em inovação de processo de software nos últimos anos, propõe- se uma caracterização através das seguintes questões secundárias:

(i) Qual etapa do processo de inovação é estudada ou/e apoiada?

(ii) Que tipo de estudo é realizado em inovação de processo de software?

(iii) Que tipo de apoio é fornecido para a inovação de processo de software?

\section{$\underline{\text { Escopo }}$}

Para delinear o escopo da pesquisa foram estabelecidos critérios para seleção das fontes de pesquisa buscando tornar a execução do estudo viável considerando custo, esforço e tempo, e garantir a acessibilidade aos dados e abrangência do estudo. Para esta seleção, os seguintes critérios foram adotados:

(i) Possuir engenho de busca que permita o uso de expressões lógicas ou mecanismo equivalente e que permita a busca no texto completo das publicações;

(ii) Pertencer a uma das editoras listadas no Portal de Periódicos da CAPES;

(iii) Incluir em sua base publicações da área de exatas ou correlatas que possuam relação direta com o tema a ser pesquisado. 


\section{Procedimento para Seleção das Publicações}

As publicações foram selecionadas em três etapas:

- $1^{\text {a }}$ Etapa: Seleção e catalogação preliminar das publicações, feita a partir da aplicação dos seguintes critérios de busca às fontes:

(i) Período: a partir de 01 de janeiro de 2000;

(ii) Idioma: inglês;

(iii) Expressão de busca (apresentado no formato da máquina de busca Scopus $\left.^{1}\right)$ :

TITLE-ABS-KEY(

(innovation $O R$ innovations $O R$ "innovative technologies" $O R$ "innovative techniques" OR "innovative practices" OR "innovative methodologies" OR "innovative methods") software))

AND ("software process" OR "software development process" OR (spi AND

) AND PUBYEAR AFT 1999

- $2^{\text {a }}$ Etapa: Como a seleção das publicações através de critérios busca não garante que todas as publicações selecionadas sejam úteis no contexto da pesquisa, por se restringir ao aspecto sintático, uma seleção das publicações relevantes é necessária. Os resumos (abstracts) foram lidos e analisados seguindo os seguintes critérios de inclusão:

(i) CI 1: A publicação descreve estudos na área de inovação de processo de software?

(ii) CI 2: A publicação realiza algum estudo ou contribuição que apoie a inovação de processo de software em alguma de suas etapas?

Cada publicação foi selecionada para a próxima etapa somente se atendesse a todos os critérios de inclusão descritos. Para diminuir o risco de que uma publicação fosse excluída prematuramente, em caso de dúvida ou não existência de resumo, a publicação não foi excluída.

- $3^{\text {a }}$ Etapa: Apesar de limitar o universo de busca, o filtro aplicado na $2^{\mathrm{a}}$ etapa não garante que todo o material coletado seja útil no contexto da pesquisa, uma vez que a seleção das publicações considerou a análise apenas do resumo da publicação. Assim, as publicações selecionadas na $2^{\mathrm{a}}$ etapa foram lidas $\mathrm{e}$ analisadas com relação ao atendimento dos critérios de inclusão definidos.

Procedimento para Extração e Armazenamento dos Dados

Para cada publicação aprovada pela expressão de busca ( $1^{\mathrm{a}}$ etapa do procedimento para seleção das publicações), os seguintes dados foram extraídos e armazenados: título, autores, ano da publicação, referência completa e resumo (abstract) da publicação.

\footnotetext{
${ }^{1}$ http://www.scopus.com
} 
Cada publicação armazenada foi examinada e submetida aos filtros de inclusão das duas etapas seguintes. Para algumas publicações o texto completo não estava disponível para acesso através das bibliotecas digitais. Neste caso, foi feita uma busca em outros meios de acesso ao conteúdo completo da publicação e quando, mesmo assim, não foi possível obter o texto completo, esta informação ficou registrada.

Para cada publicação selecionada na $3^{\mathrm{a}}$ etapa, foram respondidas as questões secundárias, registrando o tipo de estudo realizado e o tipo de apoio fornecido para a inovação de processo de software.

\section{Procedimento para Análise dos Dados}

A partir dos dados armazenados das publicações selecionadas na $3^{\mathrm{a}}$ etapa do procedimento para seleção das publicações, será realizada uma análise quantitativa que consiste em fornecer:

(i) Número de publicações selecionadas na $1^{\text {a }}$ etapa do procedimento para seleção das publicações;

(ii) Número de publicações selecionadas na $3^{\mathrm{a}}$ etapa do procedimento para seleção das publicações.

A análise qualitativa utiliza os dados da análise quantitativa e as questões secundárias de pesquisa respondidas para realizar considerações com o intuito de discutir os achados do estudo.

\subsection{Resultados}

Antes da definição da expressão de busca apresentada na $1^{\mathrm{a}}$ etapa do procedimento para seleção das publicações, alguns testes foram conduzidos de forma a tentar garantir que a expressão de busca escolhida estivesse de acordo com o objetivo e questão do estudo, com o auxílio de artigos selecionados previamente para compor o grupo de controle. Quatro rodadas de testes foram necessárias, gerando evoluções da expressão de busca. Dos 91 artigos encontrados na busca, 22 realizam algum estudo ou fornecem algum apoio ou contribuição na área de inovação de processos de software. A lista com os artigos e as informações registradas durante a realização da pesquisa podem ser encontradas em [Cerdeiral, 2011].

A distribuição dos 91 artigos encontrados na busca e dos 22 artigos selecionados dentre as máquinas de busca pode ser observada na figura 2. Apesar de todos os artigos encontrados pela Ei Compendex ${ }^{2}$ terem sido retornados pela Scopus também, os artigos retornados pela Ei Compendex foram mais relevantes.
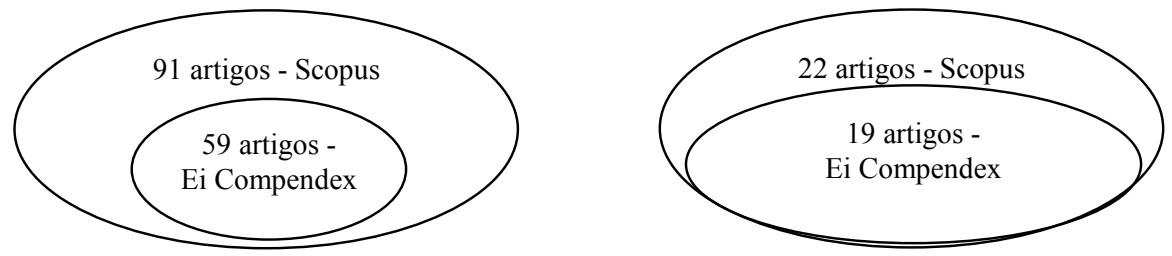

Figura 2. Distribuição dos artigos nas máquinas de busca

\footnotetext{
${ }^{2}$ http:/ / www.engineeringvillage2.org
} 
Em relação à questão secundária "Qual etapa do processo de inovação é estudada ou/e apoiada?" pode-se observar na tabela 1 que a maior parte dos artigos foca na adoção de inovações pelos profissionais da organização, buscando identificar os fatores que influenciam na adoção de inovações pelos profissionais.

Tabela 1. Quantidade de artigos por etapas do processo de inovação

\begin{tabular}{|l|c|}
\hline \multicolumn{1}{|c|}{ Etapa do processo de inovação } & Quantidade de artigos \\
\hline Transferência de conhecimento para inovação & 1 \\
\hline Geração de inovação & 3 \\
\hline Avaliação da adequação da inovação & 3 \\
\hline Adoção de inovações pelos profissionais & 11 \\
\hline Gerência do processo para a inovação & 4 \\
\hline
\end{tabular}

Em relação à questão secundária "Que tipo de estudo é realizado em inovação de processo de software?" pode-se observar na tabela 2 que, como esperado pela distribuição dos artigos dentre as etapas do processo de inovação, a maior parte são estudos do tipo survey buscando identificar os fatores que influenciam na adoção de inovações pelos profissionais em estudos de caso da adoção de alguma inovação em particular.

Tabela 2. Quantidade de artigos por tipo de estudo realizado

\begin{tabular}{|l|c|}
\hline \multicolumn{1}{|c|}{ Tipo de estudo realizado } & Quantidade de artigos \\
\hline $\begin{array}{l}\text { Estudo experimental para avaliar diferentes formas de transmissão de } \\
\text { conhecimento }\end{array}$ & 2 \\
\hline $\begin{array}{l}\text { Survey em estudos de caso da adoção de uma inovação por uma ou mais } \\
\text { organizações }\end{array}$ & 9 \\
\hline $\begin{array}{l}\text { Survey realizado em várias organizações ao redor do mundo sobre processos } \\
\text { de software }\end{array}$ & 1 \\
\hline Observações da condução de estudos de caso de implantação de inovações & 3 \\
\hline $\begin{array}{l}\text { Apenas uma comparação das funcionalidades presentes nos apoios } \\
\text { ferramentais disponíveis }\end{array}$ & 1 \\
\hline
\end{tabular}

Em relação à questão secundária "Que tipo de apoio é fornecido para a inovação de processo de software?" pode-se observar na tabela 3 que, como esperado pela distribuição dos artigos dentre as etapas do processo de inovação, a maior parte são modelos comportamentais de adoção de inovações pelos profissionais.

Tabela 3. Quantidade de artigos por apoio fornecido

\begin{tabular}{|l|c|}
\hline \multicolumn{1}{|c|}{ Apoio fornecido } & Quantidade de artigos \\
\hline $\begin{array}{l}\text { Modelos ou frameworks comportamentais de adoção de inovações pelos } \\
\text { profissionais }\end{array}$ & 9 \\
\hline Pacote de conhecimento para facilitar a transferência de conhecimento & 1 \\
\hline $\begin{array}{l}\text { Apoio ferramental para a gerência de processos de software incluindo } \\
\text { melhorias }\end{array}$ & 2 \\
\hline Definição de processos para apoiar a inovação & 2 \\
\hline Abordagens para avaliar a adequação de uma inovação & 2 \\
\hline
\end{tabular}

Com relação à questão de pesquisa "Quais são os estudos e apoios ou contribuições realizadas em inovação de processo de software nos últimos anos?", podese observar, a partir das questões secundárias de pesquisa respondidas, que apesar de existirem várias técnicas e métodos de geração de ideias nas áreas de inovação e criatividade [King e Schlicksupp, 2002; Rantanen e Domb, 2002], não há relatos de estudos da aplicação destas técnicas na área de Engenharia de Software para a inovação de processo e de tecnologia. 
Pouca atenção tem sido dada para a pesquisa proativa por inovações nos ambientes interno e externo à organização. No modelo de análise de maturidade em inovação definido por Narayana [2005] existe uma área de processo chamada "Processo para rastrear as tecnologias" que evidencia a importância desta etapa, porém nenhum apoio é fornecido para auxiliar na busca. Ardimento et al. [2008] procuram estruturar o conhecimento encontrado na literatura num pacote de conhecimento visando auxiliar na transferência de conhecimento entre a academia e a indústria, porém não auxilia na busca por esta informação pelas organizações.

A aquisição de uma inovação pelas organizações nem sempre é seguida da implantação e utilização desta inovação. A decisão de adotar uma inovação de processos ou de tecnologia normalmente parte da alta gerência da organização, que acredita que os colaboradores, com o treinamento necessário, irão adotá-la. Na prática, porém, vários esforços demonstram que aprender a utilizar uma inovação não é suficiente para sua utilização na organização, existindo fatores que influenciam a aceitação das inovações pelos profissionais. A maior parte dos estudos identificados aborda os fatores que influenciam na adoção de uma inovação com sucesso por uma organização, sendo que os fatores mais recorrentes estão relacionados com a percepção dos profíssionais das características da inovação a ser adotada e das características do ambiente e do contexto encontrado na organização [Agarwal e Prasad, 2000; Green e Hevner, 2000; Kautz e Nielsen, 2000; Cho e Kim, 2001; Rifkin, 2001; Riemenschneider et al., 2002; Gallivan, 2003; Gallivan, 2004; Green et al., 2004; Kautz e Nielsen, 2004; Green et al., 2005].

A análise e avaliação da inovação a ser adotada são enfatizadas nos processos definidos por Levine [2001] e Lind [2006]. Alguns estudos procuram aplicar os fatores que influenciam na adoção de uma inovação na avaliação de uma determinada inovação pela organização [Tortorella e Visaggio, 2001; Woo et al., 2006b; Santone e Tortorella, 2009], porém não levam em consideração grande parte dos fatores identificados, realizando uma avaliação da adequação da inovação ao processo encontrado na organização com relação as suas entradas e saídas.

Nenhum estudo identificado apoia a análise de custos e benefícios que podem ser obtidos com a implantação de uma inovação, embora o pacote de dados definido por Ardimento et al. [2008] procure estruturar as informações relacionadas a custos e benefícios encontradas na literatura. A análise de riscos relacionados com a implantação de uma inovação também não foi apoiada pelos estudos identificados.

\section{Portal Kaino ${ }^{3}$}

Os resultados do estudo baseado em revisão sistemática descritos na seção anterior demonstram que existem várias questões em aberto relacionadas com as etapas do processo de inovação, dentre elas:

- Como auxiliar na geração de ideias de melhorias inovadoras de processo e de tecnologia?

- Como monitorar as inovações tecnológicas e de processo existentes no ambiente externo à organização?

\footnotetext{
${ }^{3}$ Nome derivado de kainotomia, que em grego significa inovação e abrir novos caminhos.
} 
Buscando contribuir com estas duas questões, foi desenvolvido um ambiente no formato de portal que visa auxiliar na transferência de conhecimento e aproximação entre a academia e a indústria, além de fornecer informações de apoio para a inovação no desenvolvimento de software.

Objetivando auxiliar na primeira questão de pesquisa em aberto, são disponibilizadas no portal informações sobre várias técnicas e métodos de criatividade que apoiam diversas etapas do processo criativo [King e Schlicksupp, 2002; Rantanen e Domb, 2002], e que apesar de utilizadas nas áreas de engenharia e inovação, não tiveram seu uso na área de Engenharia de Software relatado. Para cada técnica ou método de criatividade, são descritos uma introdução e os passos necessários para a sua aplicação e um template é fornecido para apoiar a sua utilização. Além destas informações, o portal permite que algumas características da equipe que utilizará a técnica ou método sejam levadas em consideração para guiar a escolha da técnica ou método mais adequado, conforme pode ser observado na figura 3.

\begin{tabular}{|c|c|c|}
\hline \multicolumn{3}{|c|}{$\begin{array}{l}\text { Algumas ferramentas que podem auxiliar na geração de ideias inovadoras. } \\
\text { Características da Equipe }\end{array}$} \\
\hline \multicolumn{3}{|c|}{ Selecione as características da equipe que irá utilizar as ferramentas e veja quais são as mais adequadas ao seu contextc } \\
\hline \multirow{2}{*}{$\begin{array}{l}\text { Tamanho } \\
\text { Possui pouco convívio? }\end{array}$} & $\checkmark$ & Possui tempo limitado? \\
\hline & \multicolumn{2}{|r|}{ Possui tensões? } \\
\hline Possui conhecimento limitado das ferramentas? & \multirow{3}{*}{\multicolumn{2}{|c|}{ Possui conhecimento limitado do domínio? }} \\
\hline Necessita identificar o autor da ideia? & & \\
\hline Ferramentas Sugeridas & & \\
\hline Associações e Analogia Palavra-Figura & \multicolumn{2}{|c|}{ Brainstorming Imaginário } \\
\hline $\begin{array}{l}\text { A técnica é baseada nos estudos realizados em } 1961 \\
\text { por Gordon, e é reconhecida por expandir a geração } \\
\text { de ideias através da utilização de figuras, palavras e } \\
\text { biotécnicas aleatórias e aparentemente ... } \\
q\end{array}$ & \multicolumn{2}{|c|}{$\begin{array}{l}\text { A técnica proporciona a uma equipe a oportunidade } \\
\text { de 'sair do problema real', gerando ideias para um } \\
\text { problema imaginário totalmente diferente do } \\
\text { problema real, porém relacionado, e aplicando as } \\
\text { no... } \\
\qquad\end{array}$} \\
\hline Redefinição Heurística & \multicolumn{2}{|c|}{ Brainwriting 6-3-5 } \\
\hline $\begin{array}{l}\text { A técnica libera a equipe da visão fixa de que existe } \\
\text { apenas uma solução para um determinado problema, } \\
\text { permitindo que se escolha uma solução que propicie } \\
\text { um efeito melhor com menos esforço. } \\
\text { Q }\end{array}$ & \multicolumn{2}{|c|}{$\begin{array}{l}\text { A técnica foi desenvolvida por Rohrback em } 1969 \\
\text { com base no brainstorming clássico e tem como } \\
\text { objetivo a externalização de ideias na forma escrita, } \\
\text { através do seu compartilhamento em silêncio. A t... } \\
Q\end{array}$} \\
\hline
\end{tabular}

Figura 3. Informações sobre técnicas e métodos para a geração de ideias

Para auxiliar na segunda questão em aberto, o portal possui áreas nas quais os pesquisadores podem divulgar seus trabalhos de pesquisa em um formato similar ao proposto no pacote de dados de Ardimento et al. [2008], que se mostrou mais eficaz na transmissão de conhecimento. $\mathrm{O}$ objetivo destas áreas é permitir uma maior aproximação entre a academia e a indústria através da descrição dos trabalhos de pesquisa em um formato mais adequado para a indústria que o formato dos artigos e relatos técnicos.

O portal reúne informações que podem auxiliar as organizações a encontrarem os pesquisadores que mais contribuem nas áreas que se relacionam com os seus 
processos críticos, permitindo que as organizações possam acompanhar seus trabalhos de pesquisa e possam buscar por parcerias. Além dos pesquisadores, as organizações podem identificar livros, revistas e artigos mais relevantes nas áreas que se relacionam com os seus processos críticos, guiando a busca pelo conhecimento sobre inovações que possam ser adotadas. Os congressos, workshops e eventos em geral nos quais são apresentados os trabalhos mais relevantes nas áreas que se relacionam com os seus processos críticos também podem ser identificados, estimulando uma maior participação por parte da indústria nestes eventos. Por último, o portal fornece informações sobre patentes na área de software que podem auxiliar as organizações que inovam.

O portal possui dois tipos de usuários: as organizações da indústria e os pesquisadores da academia. Os autores esperam que o portal possa contribuir com ambos, auxiliando em sua aproximação e estimulando parcerias nas quais a academia possa realizar experimentos, obter dados e fomento para nutrir suas pesquisas e a indústria possa explicitar suas necessidades, contribuir e extrair benefícios dos resultados obtidos pela academia.

\section{Considerações Finais}

Este trabalho apresentou os resultados de um estudo baseado em revisão sistemática realizado para identificar o estado da arte em inovações de processo e tecnologia na área de Engenharia de Software (seção 2). Com base nos resultados do estudo, foram identificadas as etapas do processo de gerência de melhorias inovadoras e incrementais que necessitam de mais pesquisas e contribuições. Para auxiliar em algumas destas etapas, foi apresentado um ambiente no formato de portal que visa auxiliar na transferência de conhecimento e aproximação entre a academia e a indústria, além de fornecer informações de apoio para a inovação no desenvolvimento de software (seção 3). Os autores esperam que a apresentação do ambiente e de seus objetivos estimule a utilização deste pela academia, provendo informações adicionais sobre inovações realizadas na academia para a indústria.

\section{Referências}

Agarwal, R., Prasad, J. (2000) "A field study of the adoption of software process innovations by information systems professionals", Engineering Management, IEEE Transactions on, v. 47, n. 3, pp. 295-308.

Ardimento, P., Baldassarre, M.T., Cimitile, M., et al. (2008) "Empirical experimentation for validating the usability of knowledge packages in transferring innovations". In: 2nd International Conference on Software and Data Technologies, ICSOFT 2007, July 22, 2007 - July 25, 2007, v. 22 CCIS, pp. 357-370, Barcelona, Spain.

Astebro, T. (2002) "Noncapital investment costs and the adoption of CAD and CNC in U.S. metalworking industries", RAND Journal of Economics, v. 33, pp. 672688.

Aversano, L., Bodhuin, T., Canfora, G., et al. (2005) "Technology-driven business evolution", Journal of Systems and Software, v. 79, n. 3, pp. 314-338. 
Basili, V., Caldiera, G., Rombach, H. (1994) "Goal Question Metric Paradigm", Encyclopedia of Software Engineering, v. 1, pp. 528-532.

Biolchini, J., Mian, P.G., Natali, A.C. (2005) Systematic Review in Software Engineering, RT-ES 679/05, COPPE/UFRJ, Rio de Janeiro, Brasil.

Brad, S., Fulea, M., Mocan, B., et al. (2008) "Software platform for supporting open innovation". In: Automation, Quality and Testing, Robotics, 2008. AQTR 2008. IEEE International Conference on, v. 3, pp. 224-229.

Cerdeiral, C.T. (2011) Inovações Tecnológicas e de Processo em Organizações de Software, Exame de Qualificação para o D. Sc., COPPE, UFRJ, Rio de Janeiro.

Cho, I., Kim, Y.G. (2001) "Critical factors for assimilation of object-oriented programming languages", Journal of Management Information Systems, v. 18, n. Compendex, pp. 125-156.

Damanpour, R., Gopalakrishnan, S. (2001) "The dynamics of the adoption of product and process innovations in organizations", Journal of Management Studies, v. 38 , n. 1, pp. 45-66.

den Hengst, M., Hlupic, V., Currie, W.L. (2004) "The increasing need for integrating simulation and collaboration to support change management programs". In: System Sciences, 2004. Proceedings of the 37th Annual Hawaii International Conference on, pp. 8 pp.

Gallivan, M.J. (2003) "The influence of software developers' creative style on their attitudes to and assimilation of a software process innovation", Information and Management, v. 40, n. Compendex, pp. 443-465.

Gallivan, M.J. (2004) "Examining IT professionals' adaptation to technological change: The influence of gender and personal attributes", Data Base for Advances in Information Systems, v. 35, n. 3, pp. 28-49.

Green, G.C., Collins, R.W., Hevner, A.R. (2004) "Perceived control and the diffusion of software process innovations", Journal of High Technology Management Research, v. 15, n. 1, pp. 123-144.

Green, G.C., Hevner, A.R. (2000) "Successful diffusion of innovations: guidance for software development organizations", IEEE Software, v. 17, n. Compendex, pp. 96-103.

Green, G.C., Hevner, A.R., Collins, R.W. (2005) "The impacts of quality and productivity perceptions on the use of software process improvement innovations", Information and Software Technology, v. 47, n. Compendex, pp. 543-553.

Han, K.H., Kang, J.G. (2007) "Two-stage process analysis using the process-based performance measurement framework and process simulation". In: Proceedings - 
SERA 2007: Fifth ACIS International Conference on Software Engineering Research, Management, and Applications, pp. 31-37, Busan, South Korea.

ISO/IEC (2002) "15504, Information Technology - Process Assessment. Part 1 Concepts and vocabulary; part 2 - Performing an assessment; part 3 - Guidance on performing an assessment; part 4 - Guidance on use for process improvement and process capability de-termination; and part 5 - An exemplar process assessment model.".

Kautz, K., Nielsen, P.A. (2000) "Implementing software process improvement: two cases of technology transfer". In: The 33rd Annual Hawaii International Conference on System Siences (HICSS-33), January 4, 2000 - January 7, 2000, pp. 188, Maui, USA.

Kautz, K., Nielsen, P.A. (2004) "Understanding the implementation of software process improvement innovations in software organizations", Information Systems Journal, v. 14, n. 1, pp. 3-22.

Khazanchi, S., Lewis, M.W., Boyer, K.K. (2007) "Innovation-supportive culture: The impact of organizational values on process innovation", Journal of Operations Management, v. 25, n. 4, pp. 871-884.

King, B., Schlicksupp, H. (2002) Criatividade: uma vantagem competitiva Rio de Janerio, Campus.

Koc, T., Ceylan, C. (2007) "Factors impacting the innovative capacity in large-scale companies", Technovation, v. 27, n. 3, pp. 105-114.

Kuilboer, J.P., Ashrafi, N. (2000) "Software process and product improvement: an empirical assessment", Information and Software Technology, v. 42 (1) (January 1), pp. 27-34.

Levine, L. (2001) "Integrating knowledge and processes in a learning organization", IEEE Engineering Management Review, v. 29, n. Compendex, pp. 33-44.

Lind, J. (2006) "Boeing's global enterprise technology process", Research Technology Management, v. 49, n. Compendex, pp. 36-42.

Montoni, M. (2007) Uma Abordagem para Condução de Iniciativas de Melhoria de Processos de Software, Exame de Qualificação para o D. Sc., COPPE, UFRJ, Rio de Janeiro.

Narayana, M.G.P.L. (2005) "A framework approach to measure innovation maturity". In: IEMC 2005: 2005 IEEE International Engineering Management Conference, September 11, 2005 - September 13, 2005, v. II, pp. 765-769, St. John's, Newfoundland, Canada.

OECD, Eurostat (2005) "Oslo Manual: The Measurement of Scientific and Technological Activities. Proposed Guidelines for Collecting and Interpreting Innovation Data", OECD Publishing. 
Rantanen, K., Domb, E. (2002) Simplified TRIZ: New Problem-Solving Applications for Engineers and Manufacturing Professionals Florida, CRC Press.

Riemenschneider, C.K., Hardgrave, B.C., Davis, F.D. (2002) "Explaining software developer acceptance of methodologies: A comparison of five theoretical models", IEEE Transactions on Software Engineering, v. 28, n. 12, pp. 11351145 .

Rifkin, S. (2001) "Why software process innovations are not adopted", IEEE Software, v. 18 , n. Compendex, pp. $112+110-111$.

Santone, A., Tortorella, M. (2009) "Applying formal methods to process innovation". In: International Conference on Advanced Software Engineering and Its Applications, ASEA 2009, Held as Part of the Future Generation Information Technology Conference, FGIT 2009, December 10, 2009 - December 12, 2009, v. 59 CCIS, pp. 135-142, Jeju Island, Korea, Republic of.

SEI (2010) CMMI® for Development (CMMI-DEV) - Improving processs for developing better products and services, V 1.3, CMU/SEI-2010-TR-033, Software Engineering Institute.

SOFTEX (2009) MPS.BR - Melhoria de Processo do Software Brasileiro, Guia de Implementação - Parte 7, Associação para Promoção da Excelência do Software Brasileiro - SOFTEX.

SOFTEX (2011) MPS.BR - Melhoria de Processo do Software Brasileiro, Guia Geral, Associação para Promoção da Excelência do Software Brasileiro - SOFTEX.

Straub, D.W., Watson, R.T. (2001) "Research Commentary: Transformational Issues in Researching IS and Net-enabled Organizations", Information Systems Research, v. 12 , n. 4 , pp. $337-345$.

Tidd, J., Bessant, J. (2009) Managing Innovation: Integrating Technological, Market and Organizational Change, 4th ed. Chichester, UK, John Wiley \& Sons.

Tortorella, M., Visaggio, G. (2001) "Evaluation of a scenario-based reading technique for analysing process components", Journal of Software Maintenance and Evolution, v. 13, n. Compendex, pp. 149-166.

Woo, F., Mikusauskas, R., Bartlett, D., et al. (2006a) "A framework for the effective adoption of software development methodologies", ACM, Melbourne, Florida.

Woo, F., Mikusauskas, R., Bartlett, D., et al. (2006b) "A framework for the effective adoption of software development methodologies". In: 44th Annual ACM Southeast Conference, ACMSE 2006, March 10, 2006 - March 12, 2006, v. 2006, pp. 198-203, Melbourne, FL, United states. 